\title{
From Fabrication to Characterization of 3D Organic Microresonators: A Complementary Alliance of Microfluidics and Optics
}

\author{
David Pluchon, ${ }^{1}$ Nolwenn Huby, ${ }^{1}$ Alain Moréac, ${ }^{1}$ Pascal Panizza, ${ }^{1}$ and Bruno Bêche ${ }^{1,2}$ \\ ${ }^{1}$ Institut de Physique de Rennes (IPR), Université de Rennes 1, UMR CNRS 6251, France \\ ${ }^{2}$ Institut Universitaire de France (IUF), Paris, France \\ Correspondence should be addressed to Bruno Bêche, bruno.beche@univ-rennes1.fr
}

Received 25 July 2012; Accepted 2 October 2012

Academic Editor: Andreas E. Vasdekis

Copyright (C) 2012 David Pluchon et al. This is an open access article distributed under the Creative Commons Attribution License, which permits unrestricted use, distribution, and reproduction in any medium, provided the original work is properly cited.

\begin{abstract}
This paper introduces a preview of targeted current research on organic optofluidic materials and devices devoted to 3D photonics microresonators (MRs). First, such an approach takes advantage from a significant know-how on optical simulations of 3D spherical MRs by complementary and coupled ways based on electromagnetism and quantum mechanics principle. Such simulations have allowed to preset the quantization of whispering gallery modes (WGMs) and to define a new formulation of optical caustics in global 3D MRs cavities. Leaning from these simulations, an interdisciplinary approach has been achieved by combining microfluidics techniques and thin layer processes that allowed with flow rates control the realization of 3D droplets MRs of several tens of micrometers in radii. Finally, free-space optical characterizations have been performed on such 3D polymeric MRs by judicious protocols based, respectively, on a modified Raman spectroscopy laser excitation and an adequate direct beam waist optical coupling. Spectral analysis on such 3D MRs of various sizes confirms the excitation of the expected WGMs revealing free spectral range (FSR) and caustics values close to the analytical ones.
\end{abstract}

\section{Introduction}

Integrated optics $[1,2]$ plays a key role in sensors applications and optical telecommunication systems such as waveguides, Mach-Zehnder interferometers, modulators, and wavelength filters. An increasing number of these photonic devices are based on polymers materials and are increasingly being used in sensors and telecommunications applications [3-7]. Such organic materials offer numerous advantages as large volume and low cost for mass production facilities due to liquid phase processability. For several years, optical microresonators (MRs) deserve a large attention due to their specific spectral and temporal optical properties required for a wide range of applications and studies such as filtering/demultiplexing wavelength, optical switching, biological sensing, and lasers sources [8-10]. Within such systems under resonance condition, the electromagnetic fields are strongly confined near the surface as quantified modes, sources of intense locations of energy, and so-called whispering gallery modes (WGMs). Various $2 \mathrm{D}$ and 2.5D organic shapes have been reported according to the desired functionality including disks, rings, and stadium using thin layer processes and lithography or e-beam techniques [11$15]$.

The aim of this paper is to investigate a targeted current research on organic optofluidic materials based on spherical 3D MRs. This study includes theoretical electromagnetism considerations, microfluidic mechanism principles and technologies, and integrated photonics characterizations. Such a hybrid approach allows flexible thin layer processes when combined with complex fluid systems. The theoretical aspects of electromagnetism into spherical MRs are considered in Section 2. Simulations have allowed to define the quantization of whispering gallery modes (WGMs) and to found a new formulation of optical caustics in such global 3D MRs cavities. Then we describe the whole realization of microfluidic chips so as to fabricate monodisperse organic spherical family MRs with complex fluid applied devices. 
Section 3 is devoted to the optical characterization methods with the study of the photonic resonances of WGMs into the isolated spheres in air. A modified Raman excitation set-up is presented followed by an adequate protocol based on a beam-waist optical coupling. Spectral analyses with a broadband source and an optical spectrum analyzer (OSA) have been carried out for such optofluidic MRs. Finally we conclude this section by a comparison between theoretical considerations defined in Section 1 and optical measurements on free spectral range (FSR) and caustics values.

\section{Theoretical Investigation}

Classical physics states that all vectors $(E, H)$ characterizing any electromagnetic field propagating in a homogeneous, isotropic medium without any source, like our spherical resonators, satisfy the differential equation of Helmholtz, $\left(\Delta+N^{2} k^{2}\right) \psi(r, \theta, \varphi)=0$ where $N$ and $k$ represent, respectively, the optical index and the wave vector and where $\psi$ is an arbitrary electromagnetic vector. As this equation may be separable according to each variable, one can develop the expression as follows: $\psi(r, \theta, \varphi)=U(r) \Theta(\theta) \Phi(\varphi)$. Here, our interest focuses specifically on the radial behavior of the system to determine a physical parameter called caustic. So we will not discuss the complete resolution of the electromagnetic problem; see [16]. According to the spherical properties of the system, $U(r)$ can be written as $U(r)=$ $R(r) / r$, where $R(r)$ is called the radial function depicting the radial dependence of the field. Using the variable change $\rho=$ $N_{s, o} k r$ the radial equation becomes a Riccati Bessel equation whose solutions yield $R\left(N_{s, o} k r\right)=N_{s, o} k r \times j_{l}\left(N_{s, o} k r\right)$ where $j$ is a spherical Bessel function of 1st and 2nd kind [17]. Starting from this electromagnetic basic theory, we can express an analogy with a well-known problem of quantum mechanical. The expression of the differential Riccati-Bessel equation can identify the equation of a particle with an effective mass $m$ trapped in a well potential $U(r)$ similar to the Schrödinger equation independent of time [18]:

$$
\begin{aligned}
-\Delta \Psi+\frac{2 m}{\hbar}(U(r)) \Psi= & \left(\frac{2 m}{\hbar} E\right) \Psi \Longleftrightarrow-\frac{\partial^{2} R(r)}{\partial r^{2}} \\
& +\left(V(r)+\frac{l(l+1)}{r^{2}}\right) R(r)=k^{2} R(r) .
\end{aligned}
$$

In such analogy, $V(r)=k^{2}\left(1-N^{2}\right)$ represents an effective potential function, $k^{2}$ is analogous to the energy of the system, and $\hbar / 2 m=1$. An analogous well potential $V_{l}(r) \equiv$ $U(r)$ can be defined by comparison with the expression of $U(r)$. As shown in Figure 1(a), this latter evolves along $r$ up to the index step (outside optical index $N_{o}<N_{s}$ ) at the surface $(r=a)$ that involves an energy step in the system:

$$
\begin{array}{ll}
V_{l}(r)=k^{2}\left(1-N_{s}^{2}\right)+\frac{l(l+1)}{r^{2}} & \text { for } r<a, \\
V_{l}(r)=k^{2}\left(1-N_{o}^{2}\right)+\frac{l(l+1)}{r^{2}} & \text { for } r>a .
\end{array}
$$

A particular case is encountered when the energy of the well potential is equal to that of photons $k^{2}$ (zero kinetic energy), revealing particular frontiers called caustics that determine the classical and forbidden propagation regions. Inside the sphere, this limit called $r_{1}$ is associated to a turning point of the light defining a surface of propagation assimilated to a corona (Figure $1(\mathrm{~b})$ ). Thus, classical allowed regions of propagation are delimited for $r \ni\left[r_{1} ; a\right] \cup\left[r_{2} ; \infty\right.$ [ where $r_{2}$ refers to the external caustic.

As previously mentioned, WGMs require to fulfill the constructive phase condition. Considering the Eikonal approach [19], one can define the radial phase $S_{r}$ according to the parameter $r$. In such approach, integral of light phase is realized in the internal allowed region, between the surface $r=a$ and the internal caustic $r_{1}$ :

$$
\Delta S_{r}=2 L \cdot f\left(\frac{N_{s} k a}{L}\right), \quad \text { where } f(u)=\int_{1}^{u} \sqrt{1-\frac{1}{y^{2}}} d y
$$

Here, $u=a / r_{1}=N_{s} k a /(l+1 / 2)$ and $y=N_{s} k r / L$. As the angular phase is quantified by the angular momentum $L^{2}=(l(l+1))$, the radial phase condition is satisfied being a multiple of $2 \pi$. This is satisfied using an integer called radial modal number $n$ [20]. Finally, the whole quantization of wave vector according to each modal number, $n$ and $l$, leads to an expression of $k \equiv k_{n, l}$ :

$$
k_{n, l}=\frac{(l+1 / 2)}{N_{s} a} f^{-1}\left(\frac{\pi(n-1 / 4)}{l+1 / 2}\right) .
$$

According to the well-known relation of caustics $r_{x_{l}}=(l+$ $1 / 2) /\left(k N_{x}\right)$ one can express an expanded relation taking into account these two modal numbers [21] as illustrated in Figure 1:

$$
r_{1 n, l}=\frac{a N_{s}}{f^{-1}(\pi(n-1 / 4) /(l+1 / 2))}=r_{2 n, l} \times \frac{N_{o}}{N_{s}} .
$$

\section{Microfluidic Approach for 3D MRs Fabrication}

In order to compare the above theoretical study with experimental results, spherical microresonators are fabricated using microfluidic concepts and processes. The microfluidic chip made of polydimethoxysilane (PDMS, 90\% of an elastomer and $10 \%$ heat active resin, Sylgard 184 by Dow Corning) was designed by way of a mold of SU8 photoresist using standard UV lithography. Before being sealed on a PDMS support by means of a UV plasma cleaner (Harrick), the chip was perforated throughout its thickness to form injection holes (Figure 2(a)). These latest are directly connected to $380 \mu \mathrm{m}$ diameter tubings (Fisher) to inject the fluids into the microchannels. Syringe pumps (Harvard Apparatus) were used to control the flow rates of the two fluids called dispersed and continuous phases. The latter ("cp" index) was a silicon oil (viscosity $\eta_{\mathrm{cp}}=20 \mathrm{mPa} \cdot \mathrm{s}$ ) and the dispersed one ("dp" index) the NOA 89 photosensitive polymer (Norland, viscosity $\left.\eta_{\mathrm{dp}}=15 \mathrm{mPa} \cdot \mathrm{s}\right)$. The geometrical configuration of the circuit was adapted to generate periodic trains of 


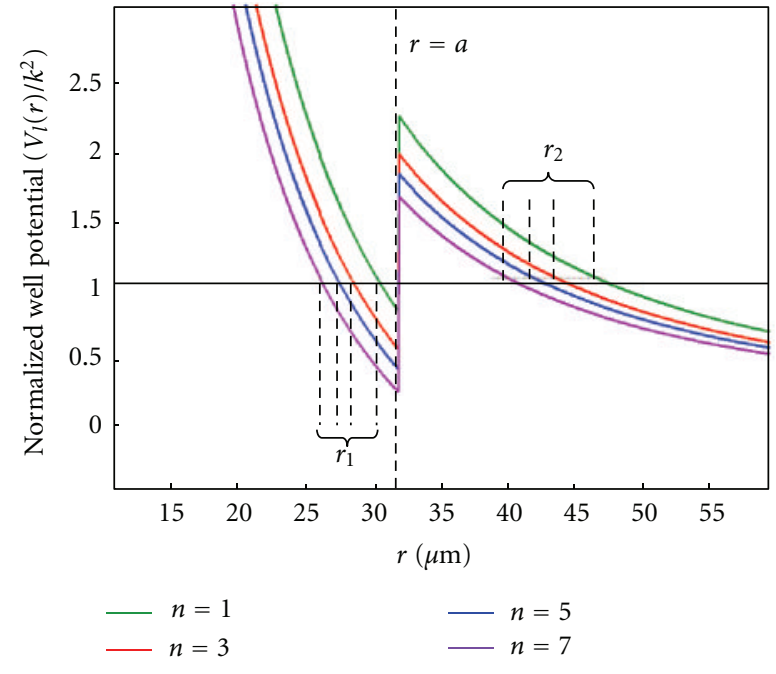

(a)

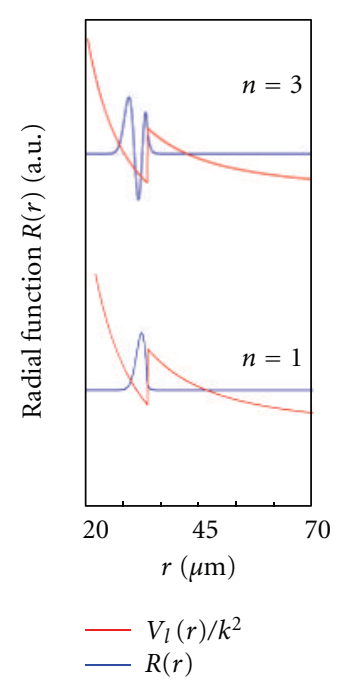

(b)

FIgURE 1: (a) Numerical simulation (cross-sectional view) of the normalized well potential $\left(V_{l}(r) / k^{2}\right)$ for various radial numbers $n=1,3$, 5, and 7. (b) Numerical simulations of the radial function $R(r)$ for $n=1$ and 3 .

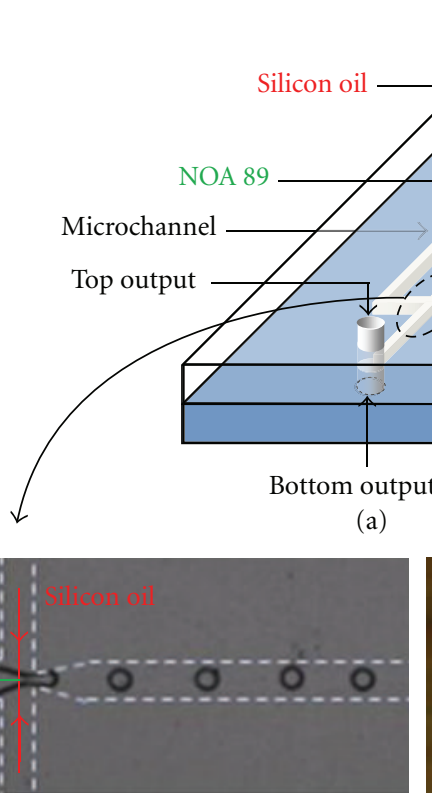

(b)

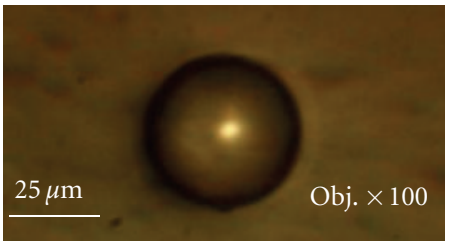

(c)

FIgURE 2: (a) Scheme of the microfluidic circuit made of PDMS. (b) Zoom on the T-junction during the fabrication of NOA 89 droplets. (c) Imaging by optical microscope of a polymerized droplet.

monodisperse droplets of tens of micrometers (Figures 2(b) and 2(c)) under flow-focusing technique [22]. In order to conserve the sphericity of droplets before polymerization, a bottom output was created (perforation of the circuit and the support) to collect droplets in a meniscus of oil. Finally, polymeric droplets were solidified by polymerization under a UV radiation at the bottom output of the microfluidic circuit.

As illustrated in Figure 2(b), the flow-focusing technique used a T-junction. This latter allows the pinching of NOA 89 polymer by the continuous phase, and the restriction (local shrinkage of the canal) placed downstream facilitates this process. At a first order, the size of droplets passing through the restriction is mainly governed by the flow rates of both phases. Such generation of spherical cavities can be divided into two steps using a straightforward model [23]. Indeed, we can first consider the blockage time ("block" index) of polymer located at the $\mathrm{T}$-junction and then the pinch time ("pinch" index) of the oil (continuous phase). According to this model and to the dimensional aspect of parameters 


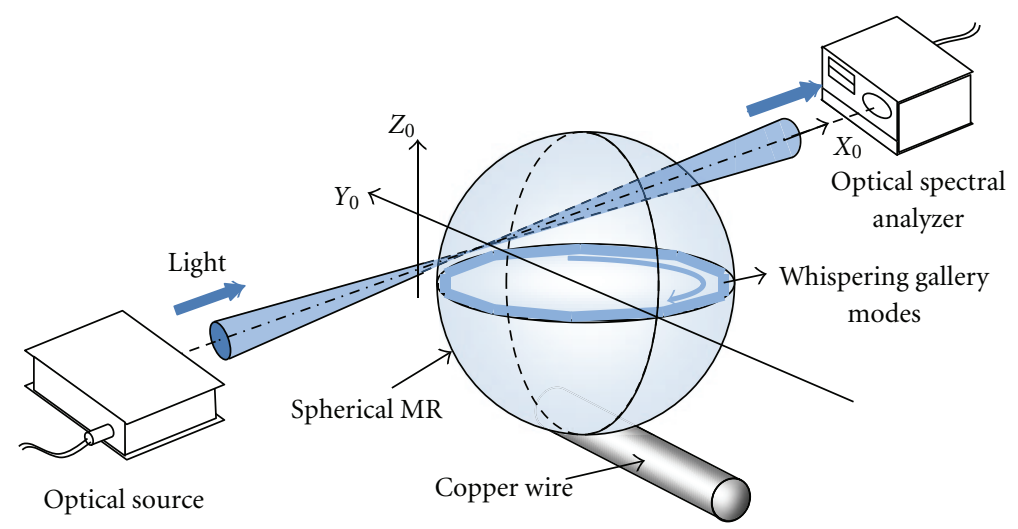

FIGURE 3: Scheme explaining the experimental set-up principle of free-space excitation used for both techniques on a microcavity positioned on a copper wire close to the beam waist region.

involved in the droplets generation, we can define a relation predicting the size of particles considering the volumes $V$ and flow rates $Q$ :

$$
V_{\text {drop }}=V_{\text {block }}+V_{\text {pinch }} \times \frac{Q_{\text {dp }}}{Q_{\mathrm{cp}}} .
$$

\section{Optical Characterizations: Results and Discussion}

4.1. Two Free-Space Methods for WGMs Highlight. The experiments presented here have been performed with the polymeric spheres (Figure 2(c)) elaborated by microfluidics technique mentioned previously. Such fabrication technique presents many advantages as a quick production, a good repeatability, and a flexibility on the dimension of structures. Moreover, produced microresonators were easy to handle and so adapted for the following optical studies performed in free space. The first is based on a micro-Raman spectrometer (HR800, Horiba Group), and the second one, called beam waist technique $[24,25]$, was performed on an optical bench to realize a spectral analysis. Whispering gallery modes propagate by total internal reflection. As illustrated in Figure 3, to enable such propagation, incident beam must be focalized close to the equator of the sphere allowing the grazing incidence of light. Such optical coupling by grazing contact leads to the diffraction of the incident beam at the edge of cavities. Assuming that cavities roughness is ideally perfect, diffraction phenomenon should not imply light scattering. However, the grazing light contact with the sphere imposes that Fresnel reflection still occurs. Such technique is so well adapted for spectral analysis in free space.

A micro-Raman spectrometer was first used for optical characterization. This latter was well adapted for a preliminary study, presenting a laser source $(\lambda=820 \mathrm{~nm})$, a spectral analyzer, and a display system. An NOA sphere $(160 \mu \mathrm{m}$ diameter) was fixed at the extremity of a thin copper tip and positioned under the laser. As shown in Figure 3, the focalized laser spot was located at the surface of the sphere allowing a grazing incidence of light beam. Also, the grazing propagation around surface was confirmed by the emergence of an intense return spot diametrically opposite (Figure 4).

Based on this free-space excitation, we realized the second study with an adapted optical bench. This latter used a light source, a spectral analyzer, and an optical microscope placed above the MRs. Thus, this optical system enabled a top view of NOA spheres and a transversal visualization during injection process. Here, beam focusing and positioning were realized using a set of optical lens and micropositioners. In a first time, the optical source was a visible blue laser $(\lambda=470 \mathrm{~nm})$ used to better appreciate the visual aspect and to follow the process of positioning leading to the TIR configuration (Figure 5). Thus, optical microscopy highlights a corona of light confinement located along the surface that confirms the TIR propagation (Figure $5(\mathrm{~d})$ ). As mentioned, such an optical characteristic is indicative of the limited propagation surface due to the inner caustic. From the theoretical study previously presented, numerical simulations were performed on such spherical cavities. Particularly, study was performed on caustics to evaluate their expansion. Thus, Figure 5(d) reveals a good agreement between the experiment and analytical expression of $r_{1}$ evaluated at $160 \mu \mathrm{m}$.

4.2. Spectral Analysis. To clearly identify WGM, optical characterizations were performed to obtain optical spectra. Indeed the observation of resonant peaks is the sign of WGMs that corresponds to light trapped in a circular orbit just within the surface of the structure by total internal reflection (TIR). They are characterized by the azimuthal modal number $l$ (integer) which is linked to the MR radius $a$ and to the light beam excitation wavelength $\lambda$ by the quantification formula: $2 \pi N_{s} a=l \cdot \lambda$ where $N_{s}$ is the optical index of spherical MRs, superior to the optical index of the surrounding media, $N_{o}$. This expression implies that circumference of cavities is an integer multiple of the wavelength. Therefore, successive resonant peaks are 


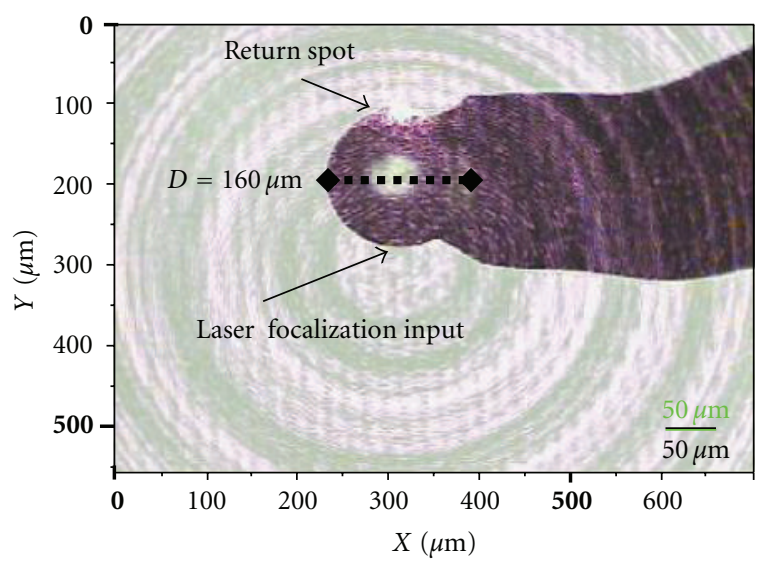

FIGURE 4: Optical microscopy image (in the direction of the laser beam) of an NOA sphere during injection on the micro-Raman set-up. The return spot of light is clearly visible after an equatorial recirculation.

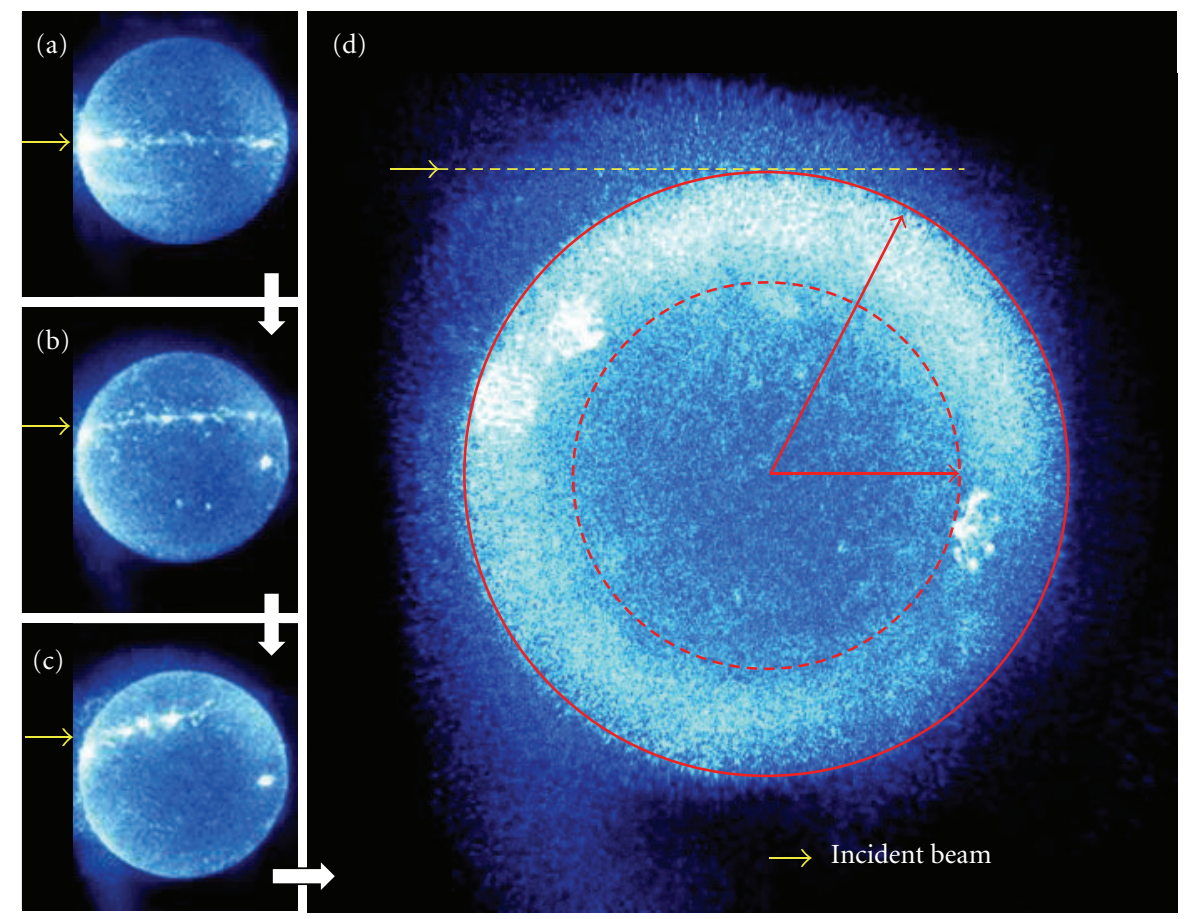

Figure 5: Top view (perpendicular to the laser beam direction) of an NOA 89 sphere $(250 \mu \mathrm{m}$ diameter $)$ during injection on the optical bench. (a) to (c) Lateral offset of the beam until (d) grazing incidence configuration. Red dashed line shows the numerical simulations of the internal caustic $r_{1}$.

regularly spaced off a specific distance called free spectral range $(\mathrm{FSR}, \Delta \lambda)$ such as

$$
\Delta \lambda=\frac{\lambda^{2}}{2 \pi N_{s} a} .
$$

Therefore, experimental determination of this parameter can confirm the WGMs propagation with calculation of MRs radius using this expression. Thus, values must match with the experimental ones (evaluated by optical microscopy) to confirm WGM.

Such spectral analyses have been performed for both excitation methods developed above. In both cases it has necessitated an accurate positioning of the laser spot in order to obtain valid resonant peaks. First, the interest of the micro-Raman investigation appears while looking closely on a shrinking wavelength range of the scattering Stockes spectrum as illustrated in Figure 6(a). One can see that spectrum presents a periodic pattern supporting the WGMs resonance in the $3 \mathrm{D} \mathrm{MR}$ and revealing an FSR estimated around $0.9 \mathrm{~nm}$. Using (7) one can estimate the diameter of the sphere around $157 \mu \mathrm{m}$, which is very close to the measurement of the sphere diameter under a microscope $(160 \mu \mathrm{m})$. On the second injection set-up, the previous blue laser was substituted by a broadband source 


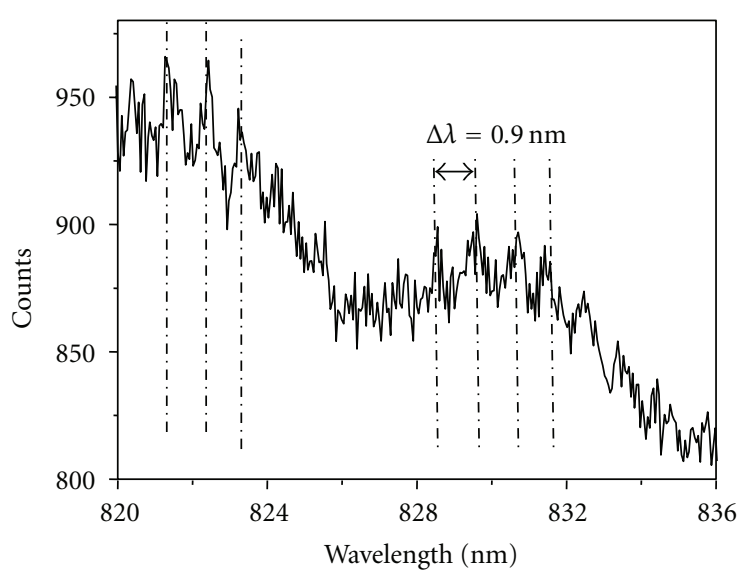

(a)

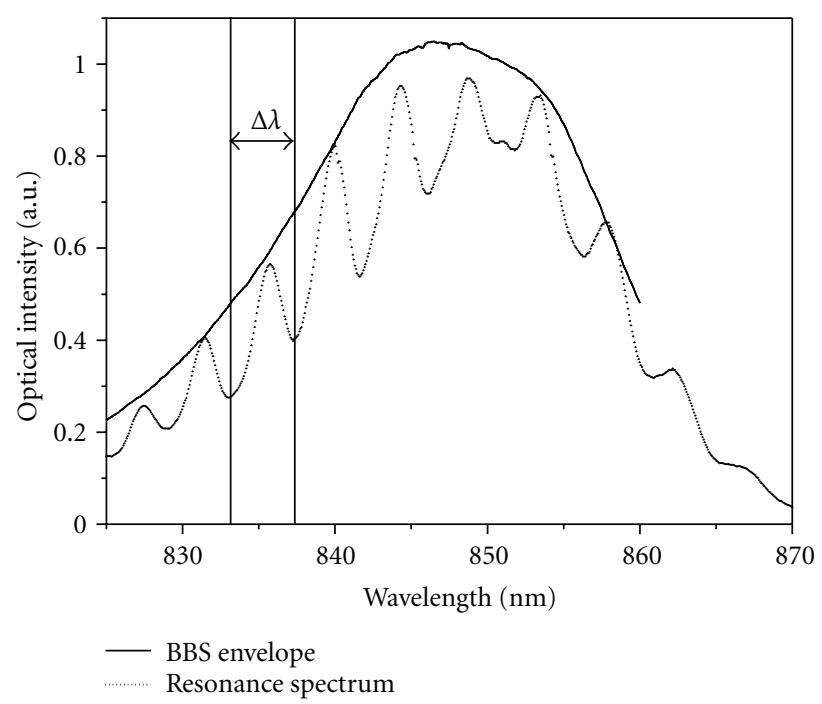

(b)

FIGURE 6: (a) Resonance spectrum of a $160 \mu \mathrm{m}$ diameter sphere excited using the micro-Raman set-up and detected with a $\times 50$ objective (FSR, $\Delta \lambda=0,9 \mathrm{~nm}$ ). (b) Resonance spectrum of a $37 \mu \mathrm{m}$ diameter sphere (FSR, $\Delta \lambda=4,17 \mathrm{~nm}$ ) and the BBS spectral envelope, recorded on the second set-up.

TABLE 1: Theoretical and experimental measurements of free spectral range (FSR) and quality factors $Q$-values for various sizes of MRs.

\begin{tabular}{|c|c|c|c|c|}
\hline$\overline{R a d i u s ~}(\mu \mathrm{m})$ & Wavelength (nm) & \multicolumn{2}{|c|}{ FSR(nm) } & $Q$ factors \\
\hline \multirow{2}{*}{18.5} & \multirow{2}{*}{847.5} & th. & 3.98 & \multirow{2}{*}{380} \\
\hline & & exp. & 4.17 & \\
\hline \multirow{2}{*}{23.5} & \multirow{2}{*}{842} & th. & 3.09 & \multirow{2}{*}{520} \\
\hline & & exp. & 3.20 & \\
\hline \multirow{2}{*}{47} & \multirow{2}{*}{847.5} & th. & 1.53 & \multirow{2}{*}{1150} \\
\hline & & exp. & 1.44 & \\
\hline
\end{tabular}

(BBS: $820 \mathrm{~nm}<\lambda<880 \mathrm{~nm}$ ) in order to observe several resonant wavelengths. Under WGM configuration, family of specific spectra as shown in Figure 6(b) was obtained. It represents the initial envelope deduced from resonant wavelengths. To confirm the veracity of such resonances, the $\Delta \lambda$-experimental free spectral range (FSR) measures were once again confronted to the theoretical ones from analytical expression (see Table 1).

The $Q$-factor is a relevant parameter that determines the optical performances of resonators. It can be expressed as the ratio of the stored energy and the energy dissipated after one cycle. The origin of $Q$-values is directly linked to three optical losses phenomenon: radiation, absorption, and scattering. However, the scattering loss appears as the main origin in the decrease of performances due to surface asperities. Experimental measurements highlight quality factors $Q=$ $\lambda /$ FWHM for such organic devices around $10^{3}$.

\section{Conclusion}

We have judiciously combined microfluidics and free-space characterization techniques for fabrication and characterization of $3 \mathrm{D}$ organic structures. The development of the microfluidic bench and processes allows a simple, rapid, and flexible fabrication of spheres with tens of microns diameters with an excellent repeatability. Free-space excitation has been used to realize a preliminary study of the resonance behavior of such optical MRs. Realization of an appropriate characterization system leads to the emergence of expected WGM resonances. First, visual aspect allowed to highlight the corona of propagation associated to the internal caustic; numerical simulations confirmed this geometrical aspect. Then, quantities summarized in Table 1 validate the good agreement between experimental and theoretical values of the radius $a$ deduced from the FSR. Finally, it appears that all these experimental, theoretical, and numerical considerations are complementary and validate all aspects going from the fabrication to the optical characterization.

\section{References}

[1] T. Tamir, Guided-Wave Optoelectronics, Springer, 1988.

[2] R. G. Hunsperger, Integrated Optics: Theory and Technology, Springer, 4th edition, 1995.

[3] P. Labbe, A. Donval, R. Hierle, E. Toussaere, and J. Zyss, "Electro-optic polymer based devices and technology for optical telecommunication," Comptes Rendus Physique, vol. 3, no. 4, pp. 543-554, 2002.

[4] F. Michelotti, A. Belardini, M. C. Larciprete et al., "Measurement of the electro-optic properties of poled polymers at 1.55 micron by means of sandwich structures with zinc oxide transparent electrode," Applied Physics Letters, vol. 83, no. 22, pp. 4477-4479, 2003.

[5] B. Bêche, N. Pelletier, E. Gaviot et al., "Conception of optical integrated circuits on polymers," Microelectronics Journal, vol. 37, no. 5, pp. 421-427, 2006.

[6] N. Pelletier, B. Bêche, L. Camberlein et al., "Single-mode rib optical waveguides on SOG/SU-8 polymer and integrated 
Mach-Zehnder for designing thermal sensors," IEEE Sensors Journal, vol. 6, no. 3, pp. 565-570, 2006.

[7] M. Ballarini, F. Frascella, N. De Leo et al., "A polymer-based functional pattern on onedimensional photonic crystals for photon sorting of fluorescence radiation," Optic Express, vol. 20, no. 6, pp. 6703-6711, 2012.

[8] A. B. Matsko, Practical Applications of Microresonators in Optics and Photonics, CRC Press, 2009.

[9] K. J. Vahala, "Optical microcavities," Nature, vol. 424, no. 6950, pp. 839-846, 2003.

[10] F. Vollmer and S. Arnold, "Whispering-gallery-mode biosensing: label-free detection down to single molecules," Nature Methods, vol. 5, no. 7, pp. 591-596, 2008.

[11] J. Scheuer and A. Yariv, "Fabrication and characterization of low-loss polymeric waveguides and micro-resonators," Journal of the European Optical Society, vol. 1, pp. 06007-1-0060075, 2006.

[12] M. Lebental, J. S. Lauret, R. Hierle, and J. Zyss, "Highly directional stadium-shaped polymer microlasers," Applied Physics Letters, vol. 88, no. 3, Article ID 031108, pp. 1-3, 2006.

[13] C. Delezoide, M. Salsac, J. Lautru et al., "Vertically coupled polymer microracetrack resonators for label-free biochemical sensors," Photonics Technolgy Letters, vol. 24, pp. 270-272, 2012.

[14] A. Zebda, L. Camberlein, B. Bêche et al., "Spin coating and plasma process for $2.5 \mathrm{D}$ integrated photonics on multilayer polymers," Thin Solid Films, vol. 516, no. 23, pp. 8668-8674, 2008.

[15] D. Pluchon, N. Huby, H. Lhermite, D. Duval, and B. Bêche, "Investigation of fabrication and resonant optical coupling in various 2D micro-resonator structures in a UV210 polymer," Journal of Micromechanics and Microengineering, vol. 22, no. 8, pp. 085016-085024, 2012.

[16] J. A. Stratton, "Electromagnetic theory," in IEEE Press Series on Electromagnetic Wave Theory, D. G. Dudley, Ed., pp. 392-420, Wiley, Hoboken, NJ, USA, 2007.

[17] M. Abramowitz and I. A. Stegun, Handbook of Mathematical Functions with Formulas, Graphs and Mathematical Tables, Dover, New York, NY, USA, 1972.

[18] B. R. Johnson, "Theory of morphology-dependent resonances: shape resonances and width formulas," Journal of the Optical Society of America A, vol. 10, no. 2, pp. 343-352, 1993.

[19] A. M. Zysk, P. S. Carney, and J. C. Schotland, "Eikonal method for calculation of coherence functions," Physical Review Letters, vol. 95, no. 4, Article ID 043904, pp. 1-4, 2005.

[20] L. Collot, V. Lefevre-Seguin, M. Brune, J. M. Raimond, and S. Haroche, "Very high-Q whispering-gallery mode resonances observed on fused silica microspheres," Europhysics Letters, vol. 23, no. 5, pp. 327-334, 1993.

[21] D. Pluchon, B. Bêche, N. Huby, and E. Gaviot, "Theoretical investigations on optical caustics of spherical microresonators: ananlytical expressions of caustics and their asymptotic behaviors, computational simulations," Optics Communications, vol. 285, pp. 2247-2254, 2012.

[22] S. L. Anna, N. Bontoux, and H. A. Stone, "Formation of dispersions using "flow focusing" in microchannels," Applied Physics Letters, vol. 82, no. 3, pp. 364-366, 2003.

[23] N. Huby, D. Pluchon, N. Coulon et al., "Design of organic 3D microresonators with microfluidics coupled to thin-film processes for photonic applications," Optics Communications, vol. 283, no. 11, pp. 2451-2456, 2010.

[24] D. Pluchon, N. Huby, A. Moréac, P. Panizza, and B. Bêche, "Flexible beam-waist technique for whispering gallery modes excitation in polymeric 3D micro-resonators," Optik. In press.
[25] D. L. Shealy and J. A. Hoffnagle, "Wavefront and caustics of a plane wave refracted by an arbitrary surface," Journal of the Optical Society of America A, vol. 25, no. 9, pp. 2370-2382, 2008. 

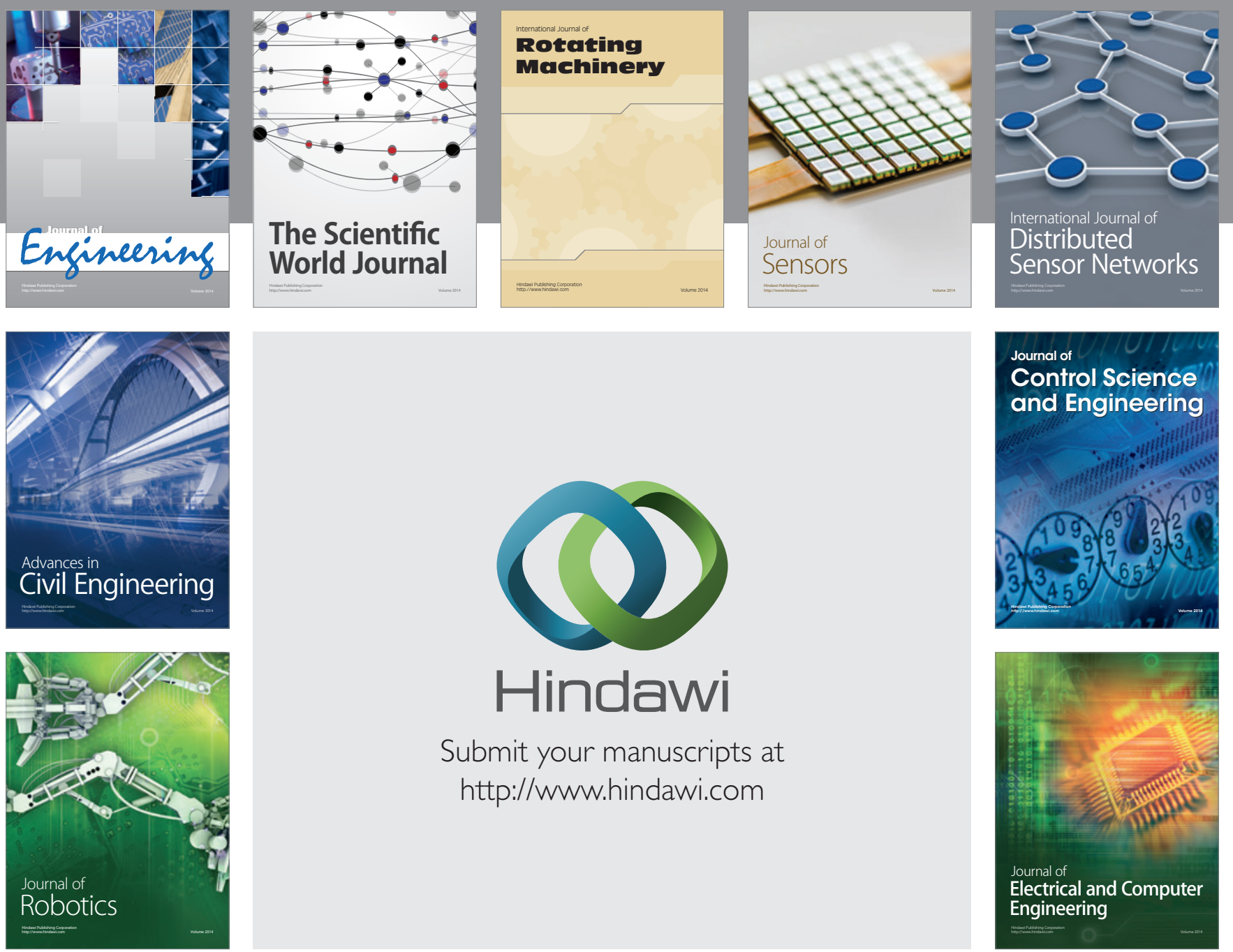

Submit your manuscripts at

http://www.hindawi.com
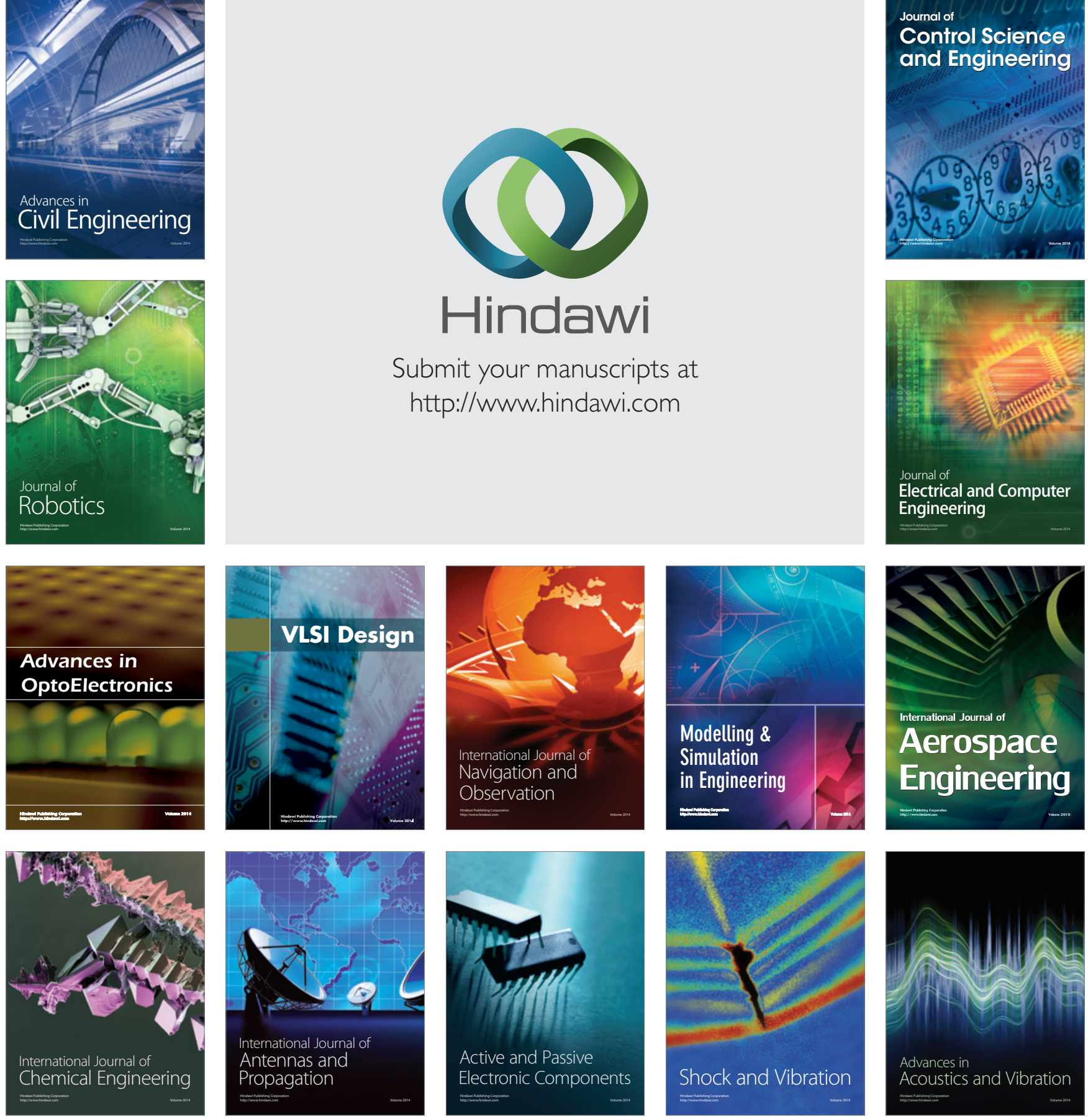